\title{
RELIABILITY IN COMPUTER NETWORKS
}

\author{
S. Minkevicius, ${ }^{1,2}$ and G. Kulvietis ${ }^{2}$ \\ ${ }_{1}^{1}$ Institute of Mathematics and Informatics, Akademijos 4, 2600 Vilnius, Lithuania, stst@ktl.mii.lt \\ ${ }^{2}$ Vilnius Gediminas Technical University, Sauletekio 11, 2040 Vilnius, Lithuania, gk@fm.vtu.lt
}

\begin{abstract}
We use a mathematical model of an open queueing network in heavy traffic. The probability limit theorem for the virtual waiting time of a customer in heavy traffic in open queueing networks has been presented. Finally, we present an application of the theorem - a reliability model from computer network practice.
\end{abstract}

keywords: mathematical models of technical systems, reliability theory, queueing theory, open queueing network, heavy traffic, the probability limit theorem, virtual waiting time of a customer.

\section{Introduction}

One can apply the theory of queueing networks to obtain probability characteristics of technical systems (for example, the reliability function of computer networks).

At first we try to present a survey of papers designated to applying the results of the queueing theory in reliability. In [2], it is investigated the reliability of a distributed program in a distributed computing system and it has been showen a probability that a program which runs on multiple processing elements that have to communicate with other processing elements for remote data files will be executed successfully. In [8], a single machine, subject to breakdown, that produces items to inventory, is considered. The main tool employed is a fluid queue model with repair. To analyze the performance of multimedia service systems, which have unreliable resources, and to estimate the capacity requirement of the systems, a capacity planning model using an open queueing network has been developed in [6]. Paper [1] discusses a novel model for a reliable system composed of $N$ unreliable systems, which can hinder or enhance one another's reliability. Paper [10] analyzes the behaviour of a heterogeneous finite-source system with a single server. As applications of this model, some problems in the field of telecommunications and reliability theory are treated. In [7] the management policy of an $M / G / 1$ queue with a single removable

Please use the following format when citing this chapter:

Author(s) [insert Last name, First-name initial(s)], 2006, in IFIP International Federation for Information Processing, Volume 199, System Modeling and Optimization, eds. Ceragioli F, Dontchev A., Furuta H., Marti K., Pandolfi L., (Boston: Springer), pp. [insert page numbers]. 
and non-reliable server is investigated. They use the analytic results of this queueing model and apply an efficient Matlab program to calculate the optimal threshold of management policy and some system characteristics. In $[3,4]$, using the law of the iterated logarithm for the queue length of customers, the reliability function of computer network is estimated and a theorem similar to Theorem 2.1 is proved.

In this paper, we present the probability limit theorem for the virtual waiting time of a customer in heavy traffic in open queueing networks.

First we consider open queueing networks with the "first come, first served" service discipline at each station and general distributions of interarrival and service time. The basic components of the queueing network are arrival processes, service processes, and routing processes. The service discipline is "first come, first served" (FCFS). We consider open queueing networks with the FCFS service discipline at each station and general distributions of interarrival and service times. The queueing network studied by us has $k$ single server stations, each of which has an associated infinite capacity waiting room. Every station has an arrival stream from outside the network, and the arrival streams are assumed to be mutually independent renewal processes. Customers are served in the order of arrival and after service they are randomly routed to either another station in the network, or out of the network entirely. Service times and routing decisions form mutually independent sequences of independent identically distributed random variables.

The basic components of the queueing network are arrival processes, service processes, and routing processes. In particular, there are mutually independent sequences of independent identically distributed random variables $\left\{z_{n}^{(j)}, n \geq 1\right\},\left\{S_{n}^{(j)}, n \geq 1\right\}$ and $\left\{\Phi_{n}^{(j)}, n \geq 1\right\}$ for $j=1,2, \ldots, k$; defined on a probability space. The random variables $z_{n}^{(j)}$ and $S_{n}^{(j)}$ are strictly positive, and $\Phi_{n}^{(j)}$ have support in $\{0,1,2, \ldots, k\}$. We define $\mu_{j}=$ $\left(M\left[S_{n}^{(j)}\right]\right)^{-1}>0, \sigma_{j}=D\left(S_{n}^{(j)}\right)>0$ and $\lambda_{j}=\left(M\left[z_{n}^{(j)}\right]\right)^{-1}>0$, $a_{j}=D\left(z_{n}^{(j)}\right)>0, j=1,2, \ldots, k$; with all of these terms assumed finite. Denote $p_{i j}=P\left(\Phi_{n}^{(i)}=j\right)>0, j=1,2, \ldots, k$. In the context of the queueing network, the random variables $z_{n}^{(j)}$ function as interarrival times (from outside the network) at the station $j$, while $S_{n}^{(j)}$ is the $n$th service time at the station $j$, and $\Phi_{n}^{(j)}$ is a routing indicator for the $n$th customer served at the station $j$. If $\Phi_{n}^{(i)}=j$ (which occurs with probability $p_{i j}$ ), then the $n$th customer served at the station $i$ is routed to the station $j$. When $\Phi_{n}^{(i)}=0$, the associated customer leaves the network. The matrix $\mathrm{P}$ is called a routing matrix. 
To construct renewal processes generated by the interarrival and service times, we assume the following for $l \geq 1, j=1,2, \ldots, k$

$$
z_{j}(0)=0, z_{j}(l)=\sum_{m=1}^{l} z_{m}^{(j)}, S_{j}(0)=0, S_{j}(l)=\sum_{m=1}^{l} S_{m}^{(j)} .
$$

Observe that this system is quite general, encompassing the tandem system, acyclic networks of $G I / G / 1$ queues, and networks of $G I / G / 1$ queues with feedback.

Let us define $V_{j}(t)$ as a virtual waiting time of a customer at the $j$ th station of the queueing network in time $t$,

$\hat{\beta}_{j}=\frac{\lambda_{j}+\sum_{i=1}^{k} \mu_{i} \cdot p_{i j}}{\mu_{j}}-1, \quad \hat{\sigma}_{j}^{2}=\frac{\sigma_{j}^{2} \cdot\left(\sum_{i=1}^{k} p_{i j}^{2}+1\right)+\left(\sum_{i=1}^{k} p_{i j}^{2} \cdot \sigma_{i}^{2}\right)+a_{j}^{2}}{\mu_{j}}$

where $j=1,2, \ldots, k$.

We suppose that the following condition is fulfilled:

$$
\lambda_{j}+\sum_{i=1}^{k} \mu_{i} \cdot p_{i j}>\mu_{j}, j=1,2, \ldots, k .
$$

Note that this condition quarantees that, with probability one there exists a virtual waiting time of a customer and this virtual waiting time of a customer is constantly growing.

One of the results of the paper is the following theorem on the probability limit theorem for the virtual waiting time of a customer in an open queueing network.

THEOREM 1 If conditions (1) are fulfilled, then

$$
\lim _{n \rightarrow \infty} P\left(\frac{V_{j}(n t)-\beta_{j} \cdot n \cdot t}{\hat{\sigma}_{j} \cdot \sqrt{n}}<x\right)=\int_{-\infty}^{x} \exp \left(-y^{2} / 2\right) d y,
$$

$0 \leq t \leq 1$ and $j=1,2, \ldots, k$.

Proof. This theorem is proved on conditions $\lambda_{j}>\mu_{j}, j=1,2, \ldots, k$ (see, for example, [9]). Applying the methods of [4], it can be proved that this theorem is true under more general (1) conditions.

The proof of the theorem is complete. 


\section{Reliability functions of the computer network}

Now we present a technical example from the computer network practice. Assume that queues arrive at a computer $v_{j}$ at the rate $\lambda_{j}$ per hour during business hours, $j=1,2, \ldots, k$. These queues are served at the rate $\mu_{j}$ per hour in the computer $v_{j}, j=1,2, \ldots, k$. After service in the computer $v_{j}$, with probability $p_{j}$ (usually $p_{j} \geq 0.9$ ), they leave the network and with probability $p_{j i}, i \neq j, 1 \leq i \leq k$ (usually $0<p_{j i} \leq 0.1$ ) arrive at the computer $v_{i}, \quad i=1,2, \ldots, k$. Also, we assume the computer $v_{j}$ fails when the virtual waiting time of queues is more than $k_{j}, j=1,2, \ldots, k$.

In this section, we will prove the following theorem on the reliability function of the computer network (probability of stopping the computer network).

THEOREM 2 If $t \geq \max _{1 \leq j \leq k} \frac{k_{j}}{\hat{\beta}_{j}}$ and conditions (1) are fulfilled, the computer network becomes unreliable (all computers fail).

Proof. At first, using Theorem 1 we get for $0<\varepsilon<1$ that

$$
\lim _{n \rightarrow \infty} P\left(\frac{V_{j}(n)-\beta_{j} \cdot n}{\hat{\sigma}_{j} \cdot \sqrt{n}}<x\right)=\int_{-\infty}^{x} \exp \left(-y^{2} / 2\right) d y, j=1,2, \ldots, k .
$$

Let us investigate a computer network which consists of the elements (computers) $v_{j}$ that are indicators of stations $X_{j}, j=1,2, \ldots, k$.

Denote

$$
X_{j}=\left\{\begin{array}{l}
1, \quad \text { if the element } v_{j} \text { is reliable, } \\
0, \quad \text { if the element } v_{j} \text { is unreliable, }
\end{array}\right.
$$

$j=1,2, \ldots, k$.

Note that $\left\{X_{j}=1\right\}=\left\{V_{j}(t)<k_{j}\right\}, j=1,2, \ldots, k$.

Denote the structural function of the system of elements connected by scheme 1 from $k$ (see, for example, [5]) as follows:

$$
\phi\left(X_{1}, X_{2}, \ldots, X_{k}\right)= \begin{cases}1, & \sum_{i=1}^{k} X_{i} \geq 1 \\ 0, & \sum_{i=1}^{k} X_{i}<1\end{cases}
$$

Let us estimate the reliability function of the computer network using the formula of full conditional probability (see [4])

$$
h\left(X_{1}, X_{2}, \ldots, X_{k}, t\right) \leq \sum_{i=1}^{k} P\left(X_{i}=1\right)
$$

Thus,

$$
0 \leq h\left(X_{1}, X_{2}, \ldots, X_{k}, t\right) \leq \sum_{i=1}^{k} P\left(V_{i}(t) \leq k_{i}\right)
$$


Applying Theorem 1 (when $t=1$ ) we obtain that

$$
\begin{gathered}
0 \leq \lim _{t \rightarrow \infty} P\left(V_{j}(t)<k_{j}\right)=\lim _{n \rightarrow \infty} P\left(V_{j}(n)<k_{j}\right)= \\
\lim _{n \rightarrow \infty} P\left(\frac{V_{j}(n)-\beta_{j} \cdot n}{\hat{\sigma}_{j} \cdot \sqrt{n}}<\frac{k_{j}-\beta_{j} \cdot n}{\hat{\sigma}_{j} \cdot \sqrt{n}}\right)=\int_{-\infty}^{-\infty} \exp \left(-y^{2} / 2\right) d y=0 .
\end{gathered}
$$

Thus (see (4)),

$$
\lim _{t \rightarrow \infty} P\left(V_{j}(t)<k_{j}\right)=0, j=1,2, \ldots, k .
$$

Consequently, $\lim _{t \rightarrow \infty} h\left(X_{1}, X_{2}, \ldots, X_{k}, t\right)=0$ (see (3) and (5)).

The proof of the theorem is complete.

Finally, we give an exact expression for $h\left(X_{1}, X_{2}, \ldots, X_{k}, t\right), t>0$. We will prove the following theorem on this probability.

THEOREM $3 h\left(X_{1}, X_{2}, \ldots, X_{k}, t\right)$ is equal to $\exp \left(-\sum_{j=1}^{k} P\left(V_{j}(t)<\right.\right.$ $\left.k_{j}\right)$ ).

Proof. First denote $\lambda_{j}, j=1,2, \ldots, k$ as intensities of structural elements, that form a complex stochastic system. Then probability of stopping this system is equal to $e^{-\sum_{j=1}^{k} \lambda_{j}}$ (see, for example, [11]).

But

$$
\lambda_{j}=M X_{j}=P\left(X_{j}=1\right)=P\left(V_{j}(t)<k_{j}\right), j=1,2, \ldots, k .
$$

Applying (6), we obtain that $h\left(X_{1}, X_{2}, \ldots, X_{k}, t\right)$ is equal to

$$
e^{-\sum_{j=1}^{k} \lambda_{j}}=e^{-\sum_{j=1}^{k} P\left(V_{j}(t)<k_{j}\right)} .
$$

The proof is complete.

As one can see, using Theorems 2 and 3, it is possible to estimate the reliability of a complex computer network.

\section{References}

[1] E. Gelenbe, J. M. Fourneau. G - networks with reset. Performance Evaluation. 49(14):179-191, 2002.

[2] M. S. Lin, D. J. Chen. The computational complexity of reliability problem on distributed systems. Information Processing Letters. 64(3):143-147, 1997.

[3] S. Minkevivcius, G. Kulvietis. On reliability in computer networks. Proceedings of International Conference of LAD' 2004. St. Petersburg, Russia, 223-229, 2004.

[4] S. Minkevivcius, G. Kulvietis. Application of the law of the iterated logarithm in open queueing networks. 2005 (to be published). 
[5] J.J. Morder, S. E. Elmaghraby. (eds.) Handbook of operational research models and applications. Van Nostrand Reinhold, New York, 1978.

[6] K. Park, S. I. Kim. A capacity planning model of unreliable multimedia service systems. Journal of Systems and Software. 63(1):69 -76, 2002.

[7] W. L. Pearn, J. C. Ke, C. Chang. Sensitivity analysis of the optimal management policy for a queueing system with a removable and non-reliable server. Computers and Industrial Engineering. 46(1):87-99, 2004.

[8] D. Perry, M. J. Posner. A correlated M/G/1-type queue with randomized server repair and maintenance modes. Operations Research Letters. 26(3):137-148, 2000.

[9] L.L. Sakalauskas, S. Minkevivcius. On the law of the iterated logarithm in open queueing networks. European Journal of Operational Research. 120:632-640, 2000.

[10] J. Sztrik, C. S. Kim. Markov-modulated finite-source queueing models in evaluation of computer and communication systems. Mathematical Modelling and Computer. 38(79):961-968, 2003.

[11] V. Zubov. (eds.) Mathematical theory of reliability of queueing systems. Radio i sviaz. 1964. 